\title{
On vertex independence number of uniform hypergraphs
}

Tariq A. CHISHTI

University of Kashmir

Department of Mathematics

Srinagar, India

email: chishtita@yahoo.co.in

\section{Shariefuddin PIRZADA}

University of Kashmir

Department of Mathematics

Srinagar, India email:

pirzadasd@kashmiruniversity.ac. in
Guofei ZHOU

Nanjing University

Department of Mathematics

Nanjing, China

email: gfzhou@mail.nju.edu.cn

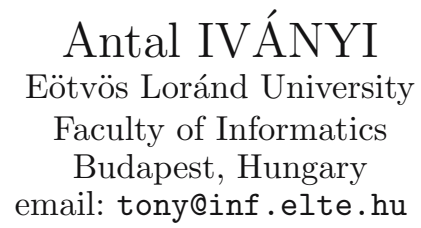

\begin{abstract}
Let $\mathrm{H}$ be an $r$-uniform hypergraph with $r \geq 2$ and let $\alpha(\mathrm{H})$ be its vertex independence number. In the paper bounds of $\alpha(H)$ are given for different uniform hypergraphs: if $H$ has no isolated vertex, then in terms of the degrees, and for triangle-free linear $\mathrm{H}$ in terms of the order and average degree.
\end{abstract}

\section{Introduction to independence in graphs}

Let $n$ be a positive integer. A graph $\mathrm{G}$ on vertex set $\mathrm{V}=\left\{v_{1}, v_{2}, \ldots, v_{n}\right\}$ is a pair $(V, E)$, where the edge set $E$ is a subset of $V \times V . n$ is the order of $G$ and $|\mathrm{E}|$ is the size of $\mathrm{G}$.

Computing Classification System 1998: G.2.2

Mathematics Subject Classification 2010: 05C30, 05C50

Key words and phrases: uniform hypergraph, independence number, lower bound DOI:10.2478/ausi-2014-0022 
Let $v \in \mathrm{V}$ and $\mathrm{N}(v)$ be the neighborhood of $v$, namely, the set of vertices $x$ so that there is an edge which contains both $v$ and $x$. Let $U$ be a subset of $\mathrm{V}$, then the subgraph of $\mathrm{G}$ induced by $\mathrm{U}$ is defined as a graph on vertex set $\mathrm{U}$ and edge set $\mathrm{E}_{\mathrm{u}}=\{(\boldsymbol{u}, \boldsymbol{v}) \mid \mathrm{u} \in \mathrm{u}$ and $\boldsymbol{v} \in \mathrm{u}\}$.

The degree $\mathrm{d}(v)$ of a vertex $v \in \mathrm{V}$ is the number of edges that contains $v$. Let $d(G)$ be the average degree of $G$, then $n d(G)=\sum_{v \in V} d(v)=2|E|$ for any graph $\mathrm{G}$. Let $\delta(\mathrm{G})$ be the minimal degree, $\Delta(\mathrm{G})$ the maximal degree of $\mathrm{G} A$ graph $\mathrm{G}$ is regular, if $\Delta(\mathrm{G})=\delta(\mathrm{G})$, and it is semi-regular, if $\Delta(\mathrm{G})-\delta(\mathrm{G})=1$.

Three vertices $v_{1}, v_{2}, v_{3}$ form a triangle in $G$ if there are distinct verticess $e_{1}, v_{2}, v_{3} \in \mathrm{F}$ such that $\left\{v_{i}, v_{i+1}\right\} \subseteq \mathrm{E}$, where the indices are taken mod 3. If $\mathrm{G}$ does not contain a triangle, then it is trianglefree.

A subset $\mathrm{U} \subseteq \mathrm{V}$ of vertices in a graph $\mathrm{G}$ is called a vertex independent set if no two vertices in $\mathrm{U}$ are adjacent. The maximum-size vertex independent set is called maximum vertex independent set. The size of the maximum vertex independent set is called vertex independence number and is denoted by $\alpha(\mathrm{G})$. The problem of finding a vertex maximum independent set and vertex independence number are NP-hard optimization problems $[73,167]$.

A maximal vertex independent set is a vertex independent set such that adding any other vertex to the set forces the set to contain an edge. The problem of finding a maximal vertex independent set can be solved in polynomial time (see e.g. the algorithms due to Tarjan and Trojanowski [155], Karp and Widgerson [101], further the improved algorithms due to Luby [128] and Alon [9].

There are exponential time exact (as Alon [9]) and polynomial time approximate algorithms (as Boppana and Haldórsson [30], Agnarsson, Haldórsson, and Losievskaja [4, 5], Losievskaja [126]) determining $\alpha(\mathrm{G})$. Also there are known algorithms producing the list of all maximum independent sets of graphs (see e.g. Johnson and Yannakakis [93], Lawler, Lenstra, Rinnooy Kan [121]).

An independent edge set of a graph $\mathrm{G}$ is a subset of the edges such that no two edges in the subset share a vertex of $G$ [166]. An independent edge set of maximum size is called a maximum independent edge set, and an independent edge set that cannot be expanded to another independent edge set by addition of any other edge in the graph is called a maximal independent edge set. The size of the largest independent edge set (i.e., of any maximum independent edge set) in a graph is known as its edge independence number (or matching number), and is denoted by $\vee(G)$. The determination of $v(G)$ is an easy task for bipartite graphs $[49,50]$, but it is a polynomially solvable problem for general graphs too $[10,101,161,162]$.

Let $\mathrm{G}=(\mathrm{V}, \mathrm{E})$ be an $\mathrm{n}$-order graph. The classical Turán theorem [159] gives 
a simple lower bound for $\alpha(G)$.

Theorem 1 (Turán [159]) If $\mathrm{n} \geq 1$ and $\mathrm{G}$ is an $\mathrm{n}$-order graph, then

$$
\alpha(G) \geq \frac{n}{d(G)+1} .
$$

This result was strengthened independently in 1979 by Caro and in 1981 by Wei.

Theorem 2 (Caro [36], Wei, [165]) If G(V,E) is a graph, then

$$
\alpha(\mathrm{G}) \geq \sum_{v \in \mathrm{V}} \frac{1}{\mathrm{~d}(v)+1} .
$$

Proof. See [36, 165].

A nice probabilistic proof of the result can be found in the paper of Alon and Spencer [11]. Since the function $\frac{1}{x+1}$ is convex, $\sum_{v \in V} \frac{1}{d(v)+1} \geq \frac{n}{d+1}$ [170].

Since this bound is the best-possible only for graphs which are unions of cliques, additional structural assumptions excluding these graphs allow improvement of 2 [80, 81]. A natural candidate for such assumptions is connectivity. In 2013 Angel, Campigotto, and Laforest [14] improved (2) for some connected graphs. For locally sparse graphs Ajtai, Erdős, Komlós and Szemerédi improved Turán's bound greatly.

Theorem 3 (Ajtai, Erdős, Komlós and Szemerédi [6, 7, 8]) If $\mathrm{G}$ is an $\mathrm{n}$-order triangle-free graph with average degree $\mathrm{d}$, then

$$
\alpha(G) \geq \frac{c n \ln d}{d+1} .
$$

Proof. See $[6,7,8]$.

They conjectured that $\mathrm{c}=1-\mathrm{o}(1)$ when $\mathrm{d}$ tends to $\infty$. Griggs [72] improved that $c$ can be $\frac{5}{12}$. Shearer [152] finally proved $c=1-o(1)$, thus confirming the conjecture. In 1994 Selkow improved the bound due to Caro and Wei supposing that the degrees of the neighbors of the vertices are also known.

Theorem 4 (Selkow [150]) If G(V,E) is a graph, then

$$
\alpha(\mathrm{G}) \geq \sum_{v \in V} \frac{1}{\mathrm{~d}(v)+1}\left(1+\max \left(0, \frac{\mathrm{d}(v)}{\mathrm{d}(v)+1}-\sum_{u \in N(v)} \frac{1}{\mathrm{~d}(\mathrm{u})+1}\right)\right) .
$$


Proof. See [150].

The bound of Selkow is equal to Caro-Wei bound for regular graph and always less then twice the Caro-Wei bound. A recent review on lower bounds for 3-order graphs was published by Henning and Yeo [89].

Let $j$ and $k$ be a positive integers. A subset $I \subseteq V(G)$ is a vertex- $k$-independent set of $\mathrm{G}$, if every vertex in $\mathrm{I}$ has at most $\mathrm{k}-1$ neighbors in $\mathrm{I}$. The vertex-k-independence number $\alpha_{k}(\mathrm{G})$ of $\mathrm{G}$ is the cardinality of the largest vertex-k-independent set of $\mathrm{G}$.

A subset $\mathrm{D} \subseteq \mathrm{V}(\mathrm{G})$ is a vertex-j-dominating set of $\mathrm{G}$, if every vertex of $\mathrm{D}$ has at least $j-1$ neighbors in $D$. The vertex- $k$-independence number $\gamma_{j}(G)$ of $\mathrm{G}$ is the cardinality of the largest vertex-j-dominating set of $\mathrm{G}$.

In 1991 Caro and Tuza [38] extended theorem of Turán to the estimation of the maximal size of k-independent sets. Thiele [156] in 1999, Csaba, Pick, and Shokoufandeh [44] in 2012 improved the bound due to Caro and Tuza. In 2008 Favoron, Hansberg and Volkmann [54] analyzed k-domination and minimum degree in graphs. Harant, Rautenbach, and Schiermeier [81, 83, 84, 85] proved different lower bounds on vertex independent number.

In 2012 Chellali and Rad [42] published a paper on k-independence critical graphs. In 2013 Caro and Hansberg [37] proposed a new approach to kindependence of graphs. Recently Chellali, Favaron, Hansberg, and Volkmann [41] published a review on k-independence.

Last year Hansberg and Pepper [79] investigated the connection between $\alpha_{k}(G)$ and $\gamma_{j}(G)$. They proved the following theorems.

Theorem 5 (Hansberg, Pepper [79]) If Let $\mathrm{G}$ be an $\mathrm{n}$-order graph, $\mathrm{j}, \mathrm{k}$ and $\mathrm{m}$ be positive integers such that $\mathrm{m}=\mathrm{j}+\mathrm{k}-1$ and let $\mathrm{H}_{\mathrm{m}}$ and $\mathrm{G}_{\mathrm{m}}$ denote, respectively, the subgraphs induced by the vertices of degree at least $\mathrm{m}$ and the vertices of degree at least $\mathrm{m}$. Then

$$
\alpha_{k}\left(H_{m}\right)+\gamma_{j}\left(G_{m}\right) \leq n
$$

and

$$
\alpha_{k}(G)+\gamma_{j}(G) \leq n\left(G_{m}\right)
$$

Proof. See [79].

Theorem 6 (Hansberg, Pepper [79]) Let G be a connected $n$-order graph with maximum degree $\Delta$ and minimum degree $\delta \geq 1$. Then

$$
\alpha_{k}(G)+\gamma_{j}(G)=n(G) \quad \text { and } \quad \alpha_{k^{\prime}}(G)+\gamma_{j^{\prime}}(G)=n(G)
$$


for every pair of integers $j, k$ and $j^{\prime}, k^{\prime}$ such that $j+k-1=\delta$ and $j^{\prime}+k^{\prime}-1=\Delta$ if and only ig $\mathrm{G}$ is regular.

Proof. See [79].

Theorem 7 (Hansberg, Pepper [79]) For any graph $\mathrm{G}$ the following two statements are equivalent:

$$
\gamma(G)+\alpha_{\delta}(G)=n(G)
$$

and

$$
\mathrm{G} \text { is regular or } \gamma(\mathrm{G})+\gamma_{2}(\mathrm{G})=\mathrm{n}(\mathrm{G}) .
$$

Proof. See [79].

Spencer [153] also published some extension of Turán theorem.

In 2014 Henning, Löwenstein, Southey and Yeo [87] proved the following theorem, which is an improvement of the result due to Fajtlowicz [53].

Theorem 8 (Henning et al. [87]) If $\mathrm{G}$ is a graph of order $\mathrm{n}$ and $\mathrm{p}$ is an integer, such that for every clique $\mathrm{X}$ in $\mathrm{G}$ there exists a vertex $\mathrm{x} \in \mathrm{X}$ such, that $\mathrm{d}(\mathrm{x})<\mathrm{p}-|\mathrm{X}|$, then $\alpha(\mathrm{G}) \geq 2 \mathrm{n} / \mathrm{p}$.

There are results on the independence number of random graphs (e.g. Balogh, Morris, Samotij [18] and Frieze [60], Henning, Löwenstein, Southey and Yeo [87], on the weighted independence number (see e.g. Halldórsson [75], Kako, Ono, Hirata, and Halldórsson [98], further Sakai, Mitsunori, and Yamazaki [149]), and on the enumeration of maximum independent sets (see e.g. Gaspers, Kratsch, and Liedloff [69].

Let $G(n, p)=(V, E)$ the random graph with vertex set $V=\left\{v_{1}, \ldots, v_{n}\right\}, p$, $\alpha\left(G_{n, p}\right)$ denote the independence number of $G_{n, p}$. In 1990 Frieze [60] proved, that if $d=n p$ and $\epsilon>0$ is fixed, then with probability going to 1 as $n \rightarrow \infty$

$$
\left|\alpha\left(G_{n}, p\right)-\frac{2 n(\ln d-\ln \ln d-\ln 2+1)}{d}\right| \leq \frac{\epsilon n}{d},
$$

provided $d_{\epsilon} \leq d=o(n)$, where $d_{\epsilon}$ is some fixed constant and $p$ is the join probability for each edge to be included in $\mathrm{E}$.

In 1983 Shearer proved the following lower bound.

Theorem 9 (Shearer [152]) If $\mathrm{G}$ is triangle-free, then

$$
\alpha(G) \geq n f(d),
$$


where

$$
f(x)=\frac{x \ln x-x+1}{(x-1)^{2}}
$$

$f(0)=1$ and $f(1)=\frac{1}{2}$.

According to the proof of Shearer for $0<x<\infty$ hold $\left.0<\mathrm{f}(\mathrm{d})<1, \mathrm{f}^{\prime} \mathrm{d}\right)<0$ and $f^{\prime \prime}(d)<0$. Further $f(x)$ satisfies the differential equation

$$
(x+1) f(x)=(x+1) d^{2} f^{\prime}(x) .
$$

It is easy to see that

$$
\lim _{x \rightarrow \infty} \frac{f(x)}{x}=\frac{\ln x}{x} .
$$

In 1995 Füredi [62] determined the number of different vertex maximal independent set in path graphs.

It is known [22] a minimum covering set of $G$ is also a maximum vertex independent set of $\mathrm{G}$. Therefore we are interested in the results on dominating sets (see e.g. [41, 54, 79, 82, 143].

The structure of the paper is as follows. After this introduction in Section 2 we present a review of results connected with th vertex and edge independence number of hypergraphs, then in Section 3 a lower bound of $\alpha(\mathrm{H})$ is presented for $n$-order $r$-uniform hipergraphs with average degree $d(H)$, and finally in Section 4 a similar bound is proved for hypergraphs not containing isolated vertex.

\section{Introduction to independence in hypergraphs}

Let $\mathrm{n} \geq 1$ and $\mathrm{W}=\left\{w_{1}, w_{2}, \ldots, w_{n}\right\}$ be a finite set called vertex set. A hypergraph $\mathrm{H}$ on vertex set $\mathrm{W}$ is a pair $(\mathrm{W}, \mathrm{F})$, where the edge set $\mathrm{F}$ is a family of the elements of $W$. We always assume that distinct edges are distinct as subsets. If each edge in $F$ contains exactly $r \geq 2$ vertices, then $\mathrm{H}$ is $\mathrm{r}$-uniform. So any graph $\mathrm{G}$ is a 2-uniform hypergraph.

Let $w \in W$ and $N(w)$ be the neighborhood of $w$, namely, the set of vertices $x$ so that there is an edge which contains both $w$ and $x$. Let $U$ be a subset of $\mathrm{W}$. The sub-hypergraph of $\mathrm{H}$ induced by $\mathrm{U}$ is defined as a hypergraph on vertex set $\mathrm{U}$ with edge set $\mathrm{F}_{\mathrm{U}}=\{\mathrm{f} \in \mathrm{F}: \mathrm{f} \subseteq \mathrm{U}\}$.

The degree $\mathrm{d}(\boldsymbol{w})$ of a vertex $w \in W$ is the number of edges that contain $w$. Let $\mathrm{d}(\mathrm{H})=\mathrm{d}$ be the average degree of an $\mathrm{r}$-uniform $\mathrm{H}$, then $\mathrm{nd}=$ $\sum_{w \in W} d(w)=r|F|$. 
For the simplicity we usually omit $\mathrm{G}$ and $\mathrm{H}$ as arguments of $\mathrm{d}(\mathrm{H})$ and similar notations.

A hypergraph $\mathrm{H}$ is linear, if any two edges of $\mathrm{H}$ have at most one vertex in common. Note that a graph $\mathrm{G}$ is always linear. Three vertices $w_{1}, w_{2}, w_{3}$ form a triangle in $\mathrm{H}$, if there are distinct edges $\mathrm{f}_{1}, \mathrm{f}_{2}, \mathrm{f}_{3} \in \mathrm{F}$ such that $\left\{\mathrm{f}_{i}, \mathrm{f}_{\mathrm{i}+1}\right\} \subseteq \mathrm{F}$, where the indices are taken mod 3.

A subset $\mathrm{U} \subseteq \mathrm{W}$ of vertices in a hypergraph $\mathrm{H}$ is called a vertex independent set if no two vertices in $\mathrm{U}$ are adjacent. The maximum-size vertex independent set of $\mathrm{H}$ is called maximum vertex independent set. The size of the maximum vertex independent set is called vertex independence number and is denoted by $\alpha(H)$. The problem of finding a maximum vertex -independent set and vertex independence number are NP-hard optimization problems [73, 167].

There are exponential time exact (as Alon [9], Tarjan and Trojanowski [155]) and polynomial time approximate algorithms (as Boppana and Haldórsson [30], Agnarsson, Haldórsson, and Losievskaja [4, 5], Losievskaja [126]). Also there are known algorithms producing the list of all maximum independent sets of graphs (see e.g. Johnson and Yannakakis [93], Lawler, Lenstra, Rinnooy Kan [121]) and hypergraphs (see e.g. Kelsen [107]).

A maximal vertex independent set is a vertex independent set such that adding any other vertex to the set forces the set to contain an edge. The problem of finding a maximal vertex independent set can be solved in polynomial time (see e.g. the algorithms due to Tarjan and Trojanowski [155], Karp and Widgerson [101], further the improved algorithms due to Luby [128] and Noga [9]).

In 2012 Dutta, Mubayi, and Subramanian [48] gave new lower bond for the vertex independence number of sparse hypergraphs.

In 2013 Eustis devoted a $\mathrm{PhD}$ dissertation to the problems of hypergraph independence numbers $[51,52]$.

An independent edge set of a hypergraph $\mathrm{H}$ is a subset of the edges such that no two edges in the subset share a vertex of $H$ [136]. An independent edge set of maximum size is called a maximum independent edge set, and an independent edge set that cannot be expanded to another independent edge set by addition of any other edge in the hypergraph is called a maximal independent edge set. The size of the largest independent edge set (i.e., of any maximum independent edge set) in a hypergraph is known as its edge independence number (or matching number), and is denoted by $v(\mathrm{H})$. The determination of $v(\mathrm{H})$ is an easy task for bipartite graphs [49,50], but it is a polynomially solvable problem for general graphs too [10].

There are many results on the characterization of hypergraph score se- 
quences and on their reconstruction (see e.g. [20, 110, 140, 171, 139, 164, 172]), on the enumeration of different hypergraphs (see e.g. [21, 47, 138, 144, 145]) and directed hypergraphs (see e.g. [15]).

An $\mathrm{r}$-uniform hypergraph with $\mathrm{n}$ vertices is called complete, if its set of edges has the cardinality $\left(\begin{array}{c}n \\ 2\end{array}\right)$. The complement of an r-uniform hypergraph $\mathrm{H}$ is $\overline{\mathrm{H}}=(\mathrm{W}, \overline{\mathrm{F}})$, if $|\mathrm{F} \cup \overline{\mathrm{F}}|=\left(\begin{array}{c}n \\ 2\end{array}\right)$ and $|\mathrm{F} \cap \overline{\mathrm{F}}|=0$.

A set $\mathrm{P} \subseteq \mathrm{W}$ is called an edge cover of $\mathrm{H}$, if for any non-isolated vertex $x \in W$ there exists an edge $f_{i} \in P$ that $x \in f_{i}$. The cardinality of a minimum set which is an edge covering of $\mathrm{H}$ is called the edge covering number of $\mathrm{H}$, and is denoted by $v(\mathrm{H})$.

The following lemma, proved in [97], gives a relation between the edge covering number and the edge independence number in an $r$-uniform hypergraph $\mathrm{H}$ without isolated vertices.

Lemma 10 (Jucovič, Olejník [97]) For an r-uniform n-order hypergraph $\mathrm{H}$ with $\mathrm{n}$ without isolated vertices the following inequalities hold:

$$
\begin{gathered}
\alpha(H) \leq n-(k r-1) v(H), \\
\alpha(H)+(r-1) v(H) \leq n . \\
v(H)+(r-1) r-1 v(H) \geq n,
\end{gathered}
$$

Proof. See [97].

This lemma generalizes the relations published by Gallai [67] in 1959. In 1991 Tuza [160] extended Gallai's inequalty for uniform hypergraphs.

In 1989 Olejník proved the following three theorems characterizing $\alpha(H)$ and $v(H)$.

Theorem 11 (Olejník [136]) For an r-uniform n-order hypergraph $\mathrm{H}=(\mathrm{W}, \mathrm{F})$ with $\mathrm{n}$ and its complement $\overline{\mathrm{H}}=(\mathrm{W}, \overline{\mathrm{F}})$

$$
\left\lfloor\frac{n}{r}\right\rfloor \leq v(H)+v(\bar{H}) \leq 2\left\lfloor\frac{n}{r}\right\rfloor
$$

and

$$
0 \leq v(H) v(\bar{H}) \leq\left\lfloor\frac{n}{r}\right\rfloor^{2}
$$

Proof. See [136].

This bounds are direct generalizations of the bounds published by Chartrand and Schuster in 1974 [40]. 
Theorem 12 (Olejník [136]) For an r-uniform n-order hypergraph $\mathrm{H}=(\mathrm{W}, \mathrm{F})$ and its complement $\overline{\mathrm{H}}=(\mathrm{W}, \overline{\mathrm{F}})$, where neither $\mathrm{H}$ nor $\overline{\mathrm{F}}$ have isolated vertices,

$$
\left\lfloor\frac{n}{r}\right\rfloor \leq v(H)+v(\bar{H}) \leq 2\left\lfloor\frac{n}{r}\right\rfloor
$$

and

$$
0 \leq v(H) v(\bar{H}) \leq\left\lfloor\frac{n}{r}\right\rfloor^{2}
$$

Proof. See [136].

This result is an extension of the work of R. Laskar and B. Auerbach published in 1978 [120].

Theorem 13 (Olejník [136]) For an r-uniform n-order hypergraph $\mathrm{H}=(\mathrm{W}, \mathrm{F})$ and its complement $\overline{\mathrm{H}}, \overline{\mathrm{F}}$, where neither $\mathrm{H}$ nor $\overline{\mathrm{H}}$ have isolated vertices and $\mathrm{n} \neq 2 \mathrm{r}$

$$
2\left\lfloor\frac{n}{r}\right\rfloor \leq \alpha H+\alpha \bar{H} \leq 2 n-(r-1)\left\lfloor\frac{n}{r}\right\rfloor-r+1
$$

and

$$
\left\lfloor\frac{n}{r}\right\rfloor^{2} \leq \alpha(H) \alpha(\bar{H}) \leq \frac{1}{4}\left(2 n-(r-1)\left\lfloor\frac{n}{r}\right\rfloor-k+1\right)^{2} .
$$

Proof. See [136].

In 1993 Gallo, Longo, Nguyen, and Pallottino [68] studied the applications of directed hypergraphs. In 2004 Vietri [163] wrote on the complexity of the arc-coloring of directed hypergraphs. In 2003 Frank, Király and Király [55] analized the orientation of directed hypergraphs.

Let

$$
B(p, q)=\int_{0}^{1}(1-t)^{p-1} t^{q-1} d t
$$

denote the beta-function with $p, q>0$. Set constants $0<a \leq 1,0<b \leq 1$, and $B=B(a, 1-b)$, and let

$$
f_{r}(x)=\frac{1}{B} \int_{0}^{1} \frac{1-t)^{a}}{\left(t^{b}[1+(x-1) t]\right.} d r .
$$

In 2004 Zhou and Li [170] proved the following theorem on sparse hypergraphs.

Theorem 14 (Zhou, Li [170]) Let $\mathrm{H}$ be a triangle-free, $\mathrm{r}$-uniform $(\mathrm{r} \geq 2$ ) $\mathrm{n}$-order linear hypergraph with average degree $d$. Then its strong vertex independence number $\alpha_{\mathrm{s}}(\mathrm{G})$ is at least $\mathrm{nf}_{\mathrm{r}}(\mathrm{d})$. 
Proof. See [170].

In 2004 Greenhill, Ruciński, and Wormald [71] analyzed random hypergraph processes with degree restrictions. In 2008 Plociennik [141] proposed an approximation algorithm for the vertex maximum independence set problem of uniform random hypergraphs. M. Halldórsson, and Losievskaja [4, 5] used semidefinit programming to find maximum vertex independent set of hypergraphs.

Shearer's result ([152], further (11) and (12)) was generalized in [170] with the function $g_{r}(x)$ satisfying

$$
(r-1)^{2} x(x-1) g_{r}^{\prime}(x)+[(r-1) x+1] g_{r}(x)=1
$$

for r-uniform, triangle-free linear hypergraphs, with sparse neighborhood and in [125] with the function $g_{r, m}(x)$ satisfying

$$
(r-1)^{2} x(x-m) g_{r, m}^{\prime}(x)+[(r-1) x+1] g_{r, m}(x)=1
$$

for $r$-uniform, triangle-free, and double linear hypergraphs, in which each subhypergraph induced by a neighborhood, has maximum degree less than $\mathrm{m}$. A linear hypergraph is called double linear if for any non-adjacent distinct vertices $w$ and $z$, each edge containing $w$ has at most one neighbor of $z$. From the uniqueness of solutions of the differential equations, we see that $g_{2}(x)=g(x)$ and $g_{r, 1}(x)=g_{r}(x)$. It is shown [125] that $g_{2, m}(x) \sim \frac{\log x}{x}$, and for $g_{r, m}(x) \sim \frac{c}{d^{1 /(r-1)}}$ for $r \geq 3$, where $c=c(r, m)>0$ is a constant without knowing exact values.

Independent sets and numbers are studied in many papers (see e.g. the papers of Abraham [1], Alon, Uri and Azar [12], Berger and Ziv [23], Bollobás, Daykin and Erdős [27], Bonato, Brown, Mitsche and Pralat [28, 29], Bordewich, Dyer and Karpiński [31], Boros, Gurvich, Elbassioni, Gurvich and Khachiyan [32, 33], Borowiecki and Michalak [34], Cutler and Radcliffe [45], Greenhill [70], Halldórson and Losievskaja [76], Hofmeister and Lehman [90], Johnson and Yannakakis [93], Khachiyan, Boros, Gurvich, and Elbassioni [108], Lepin [122], Li and Zhang [125], Losievskaja [126], Shachnai and Srinivasan [151], Tarjan and Trojanowski [155], Yuster [168]).

Since independence number and matching number are closely connected, we are interested in the results on maximum matching algorithms too (see e.g. $[25,26,46,47,49,50,56,57,61,65,66,77,78,86,88,89,91,92,100,104$, $105,109,112,113,118,119,127,131,132,133,135,137,142,146,147,148$, $154,157,158,169])$. 
Minimum dominating set of $\mathrm{H}$ and maximum vertex independent set of $\mathrm{H}$ are connected concepts, therefore we are interested in the results on dominating sets of hypergraphs (see e.g. $[2,96]$ ).

Further connected problems are also often analyzed (see e.g. e.g. in the papers of Agnarsson, Egilssson, and Halldórson [3], Alon, Frankl, Huan, Rödl, Ruciński [10], Alon and Yuster [13], Baranyai [19], Balogh, Butterfield, Hu and Lenz [17], Bertram-Kretzberg and Letzman [24], Bujtás and Tuza [35], Cockayne, Hedetniemi, and Laskar [43], Frank, Király and Király [55], Frankl and Rödl [58, 59], Füredi, Ruszinkó, and Selver [63, 64], Hán, Person and Schacht [78], Henning and Yeo [89], Huang, Loh and Sudakov [92], Johnson and Yannakakis [93], Johnston and $\mathrm{Lu}$ [94, 95], Jucovič and Olejník [97], Karonśki and Luczak [99], Katona [102, 103], Keevash and Sudakov [106], Kelsen [107], Kohayakawa, Rödl, Skokan [111], Krivelevich [115], Kühn and Loose [117], Kostochka, Mubayi, Verstraëte [114], Krivelevich, Nathaniel, and Sudakov [116], Li, Rousseau and Zang [123, 124], Luczak and Szymańska [129, 134], Szymańska [154], Treglown and Zhao [157, 158], Tuza [160], Yuster [169]).

Although hypergraphs are less often used in the practice than the graphs, they also have different applications in the practice.

For example Bailey, Manoukian, Ramamohanaro [16], further Gunopolus, Khardon, Mannila and Toivonen [74] reported on the applications in data mining, Gallo, Longo, Nguyen, and Pallottino [68], further and Maier [130] in relational databases.

In 2000 Carr, Lancia, Istrail, and Genomics [39] reported on Branch-andCut algorithms for vertex independent set problem and on their application to solve problems connected with protein structure alignment.

In this paper, we obtain $\alpha(H) \geq \sum_{v \in V} \frac{1-1 / r}{d(v)^{1 /(r-1)}}$ for any $r$-uniform hypergraph $\mathrm{H}$ without the condition of being triangle-free. The algorithm is naive: it deletes a vertex of maximum degree repeatedly. In order to get a large independent set, a commonly used algorithm is to find a suitable vertex $v$, then delete $v$ and its neighbors, and then do the iterations. Deleting all neighbors seems to be of no use for hypergraphs as in $[125,170]$. After deleting a vertex $v$, we delete only one vertex other than $v$ from each edge containing $v$. Our new function $f_{r}(x)$ satisfies

$$
\left[(r-1) x^{2}-x\right] f_{r}^{\prime}(x)+(x+1) f_{r}(x)=1 .
$$

Then $f_{r}(x) \sim \frac{c}{x^{1 /(r-1)}}$ as $x \rightarrow \infty$. We do not know the exact value of $c=c(r)$. However, when we run the algorithm, we note that for a vertex $v$, we delete $1+d(v)$ vertices instead of deleting $1+(r-1) d(v)$ vertices as in $[125,170]$. So 
if $\mathrm{c}$ is the constant such that $\mathrm{g}_{\mathrm{r}}(x) \sim \frac{\mathrm{c}}{\chi^{1 /(r-1)}}$ as $x \rightarrow \infty$, then the new constant seems to be $(r-1) c$, namely, $f_{r}(x) \sim \frac{(r-1) c}{\chi^{1 /(r-1)}}$.

\section{Bound for uniform hypergraphs without isolated vertex}

The following Theorem 15 is a corollary of Theorem 18, but it has an easy probabilistic proof.

Theorem 15 Let $\mathrm{H}=(\mathrm{V}, \mathrm{E})$ be an $\mathrm{r}$-uniform hypergraph of order $\mathrm{n}$ and average degree $\mathrm{d} \geq 1$, then

$$
\alpha(H) \geq\left(1-\frac{1}{r}\right) \frac{n}{d^{1 /(r-1)}} .
$$

Proof. Define a random subset $\mathrm{U} \subseteq \mathrm{V}$ by $\operatorname{Pr}(v \in \mathrm{U})=\mathrm{p}$ for some $0 \leq \mathrm{p} \leq 1$ with all these events being mutually independent over $v \in \mathrm{V}$.

Let $X(U)$ be the number of vertices in $U$ and let $Y(U)$ be the number of edges in the subgraph induced by $\mathrm{U}$. Note that for one of the edges of $\mathrm{H}$, the probability that all of its vertices belong to $\mathrm{U}$ is $\mathrm{p}^{\mathrm{r}}$. By linearity of expectation, we have

$$
E(X-Y)=E(X)-E(Y)=n p-\frac{n d}{r} p^{r} .
$$

Thus there exists a set $\mathrm{U}$ satisfying

$$
X(U)-Y(U) \geq E(X)-E(Y) .
$$

Note that $\mathrm{U}$ is not that we require, since the sub-hypergraph of $\mathrm{H}$ induced by $\mathrm{U}$ may have edges. However, if we delete one vertex from each edge contained in $U$, then at most $Y(U)$ vertices are deleted, we thus obtain a new set with at least $E(X)-E(Y)$ vertices and whose induced sub-hypergraph has no edges. The desired lower bound follows by taking $p=\frac{1}{\mathrm{~d}^{1 /(r-1)}}$.

For hypergraphs that are not regular, Theorem 18 is stronger than Theorem 15. We need two lemmas for the proof of Theorem 18.

Lemma 16 Let $\mathrm{r} \geq 2$ be an integer and define

$$
h_{r}(x)= \begin{cases}1-x / r & \text { if } 0 \leq x<1 \\ \frac{1-1 / r}{x^{1 /(r-1)}} & \text { if } x \geq 1,\end{cases}
$$

then $\mathrm{h}_{\mathrm{r}}(\mathrm{x})$ is positive, decreasing and convex. Furthermore, for $\mathrm{x} \geq 1$, the function $\mathrm{h}_{\mathrm{r}}(\mathrm{x})$ satisfies that $(\mathrm{r}-1) \mathrm{x} \mathrm{h}^{\prime}(\mathrm{x})+\mathrm{h}_{\mathrm{r}}(\mathrm{x})=0$. 
Proof. It is easy to see that $h_{r}(x)$ is positive and

$$
h_{r}^{\prime}(x)= \begin{cases}-1 / r & \text { if } 0 \leq x<1 \\ \frac{-1 / r}{x^{r /(r-1)}} & \text { if } x \geq 1\end{cases}
$$

So $h_{r}^{\prime}(x)$ is continuous, negative and increasing, thus $h_{r}(x)$ is decreasing and convex. The fact that $h_{r}(x)$ satisfies the mentioned differential equation is straightforward.

Let $\Delta=\Delta(\mathrm{H})$ denote the maximal degree in $\mathrm{H}$ and define

$$
S(G)=\sum_{x \in V} h(d(x)), \quad S(H)=\sum_{x \in W} h(d(x)) .
$$

Lemma 17 If $\Delta(\mathrm{H}) \geq 1, w \in W, \mathrm{~d}(w)=\Delta(\mathrm{H})$, and $\mathrm{H}_{1}=\mathrm{H}-\{w\}$, then $S\left(H_{1}\right) \geq S(G)$.

Proof. For each $x \in V \backslash\{v\}$, denote by $n_{x}$ the number of edges of $H$ that contain both $x$ and $v$. Then $n_{x}=0$ if $x$ and $v$ are not adjacent, and $n_{x} \geq 1$ otherwise. It is easy to see

$$
\sum_{x \in \bigvee \backslash\{v\}} n_{x}=(r-1) \Delta
$$

since $\mathrm{H}$ is $\mathrm{r}$-uniform. On the other hand, we have

$$
S\left(H_{1}\right)=S(H)-h(\Delta)+\sum_{x \in \bigvee\{v\}}\left[h\left(d(x)-n_{x}\right)-h(d(x))\right] .
$$

From the fact that $h^{\prime}(x)$ is negative and increasing, we have

$$
h\left(d(x)-n_{x}\right)-h(d(x))=-h^{\prime}\left(\theta_{x}\right) n_{x} \geq-h^{\prime}(\Delta) n_{x},
$$

where $\theta_{x} \in\left[d(x)-n_{x}, d(x)\right]$, thus

$$
\begin{aligned}
\mathrm{S}\left(\mathrm{H}_{1}\right) & \geq \mathrm{S}(\mathrm{H})-\mathrm{h}(\Delta)-\mathrm{h}^{\prime}(\Delta) \sum_{x \in \mathrm{V} \backslash\{v\}} \mathrm{n}_{\mathrm{x}} \\
& =\mathrm{S}(\mathrm{H})-\mathrm{h}(\Delta)-(\mathrm{r}-1) \Delta \mathrm{h}^{\prime}(\Delta) \\
& =\mathrm{S}(\mathrm{H}),
\end{aligned}
$$

proving the claim. 
Theorem 18 Let $\mathrm{H}=(\mathrm{V}, \mathrm{E})$ be an r-uniform hypergraph without isolated vertex, then

$$
\alpha(H) \geq\left(1-\frac{1}{r}\right) \sum_{v \in V} \frac{1}{d(v)^{1 /(r-1)}} .
$$

Proof. We write $h_{r}(x)$ as $h(x)$ for simplicity and define

$$
S(H)=\sum_{x \in V} h(d(x)) .
$$

Repeat the algorithm by deleting the vertex of maximum degree if the degree is at least one, terminate the algorithm if there are no edges. Denote by $\mathrm{H}_{0}=$ $\mathrm{H}, \mathrm{H}_{1}, \ldots, \mathrm{H}_{\ell}$ for the sequence of hypergraphs, where $\mathrm{H}_{\ell}$ has no edge. We get $S\left(H_{\ell}\right)=n-\ell$ since $h(0)=1$, where $n-\ell$ is the order of $H_{\ell}$, and $\alpha(H) \geq n-\ell$. So

$$
\alpha(H) \geq S\left(H_{\ell}\right) \geq S\left(H_{\ell-1}\right) \geq \cdots \geq S\left(H_{0}\right)=S(H),
$$

the assertion follows immediately.

Since the function $\frac{1}{x^{1 /(r-1)}}$ is convex, Theorem 15 is truly a corollary of Theorem 18.

Remark. Theorem 18 gives $\alpha(\mathrm{G}) \geq \sum_{v} \frac{1}{2 \mathrm{~d}(v)}$ for a graph $\mathrm{G}$ with $\delta(\mathrm{G}) \geq 1$, which is weaker than $\alpha(\mathrm{G}) \geq \sum_{v} \frac{1}{\mathrm{~d}(v)+1}$. However, the later can be proved similarly by replacing the function $h(x)$ with $1 /(x+1)$. For details of this algorithm, see Griggs [72].

\section{Bound for uniform linear triangle-free hypergraphs}

In this section triangle-free hypergraphs are considered. To generalize Shearer's method [152] and to delete less vertices for a hypergraph, we have a definition as follows.

Let $\mathrm{H}=(\mathrm{V}, \mathrm{E})$ be an $\mathrm{r}$-uniform hypergraph and let $v$ be a vertex of $\mathrm{H}$, denote by $E_{v}=\{e \in E: v \in e\}=\left\{e_{1}, e_{2}, \ldots, e_{d(v)}\right\}$ for the set of edges containing $v$. A claw of $v$ is a set of neighbors of $v$ of the form $\left\{u_{1}, u_{2}, \ldots, u_{d}(v)\right\}$ such that each $u_{i} \in e_{i}-v$. For a claw $T$ of $v$, we write as $Q_{T}$, the number of edges that intersect $\mathrm{T}$.

When we run the algorithm in each step, we will delete $v$ and a claw $\mathrm{T}$, so $\mathrm{Q}_{\mathrm{T}}$ edges will be deleted. The new function is as follows. 
Let $r \geq 2$ be and integer and let $b=\frac{r-2}{r-1}$. Define

$$
f_{r}(x)=\frac{1}{r-1} \int_{0}^{1} \frac{1-t}{t^{b}[1+((r-1) x-1) t]} d t .
$$

Lemma 19 The function $\mathrm{f}_{\mathrm{r}}(\mathrm{x})$ satisfies the differential equation

$$
\left[(r-1) x^{2}-x\right] f_{r}^{\prime}(x)+(x+1) f_{r}(x)=1,
$$

and it is positive, decreasing and convex.

Proof. By differentiating under the integral and then integrating by parts, we have

$$
\begin{aligned}
& {\left[(r-1) x^{2}-x\right] f_{r}^{\prime}(x) } \\
= & -\left[(r-1) x^{2}-x\right] \int_{0}^{1} \frac{1-t}{t^{1-b}[1+((r-1) x-1) t]^{2}} d t \\
= & x \int_{0}^{1}(1-t) t^{1-b} \frac{d}{d t}\left(\frac{1}{1+[(r-1) x-1] t}\right) \\
= & -x \int_{0}^{1} \frac{1}{1+[(r-1) x-1] t}\left[(1-t)(1-b) t^{-b}-t^{1-b}\right] d t \\
= & -(r-1)(1-b) x f_{r}(x)+x \int_{0}^{1} \frac{t^{1-b}}{1+[(r-1) x-1] t} d t \\
= & -x f_{r}(x)+\frac{1}{r-1} \int_{0}^{1}\left(\frac{1}{1-t}-\frac{1}{1+[(r-1) x-1] t}\right)(1-t) t^{-b} d t \\
= & -x f_{r}(x)+1-f_{r}(x) \\
= & 1-(x+1) f_{r}(x)
\end{aligned}
$$

which follows by the differential equation. The monotonicity and convexity of $f_{r}(x)$ can be seen by repeated differentiation under the integral.

Theorem 20 Let $\mathrm{H}$ be an $\mathrm{r}$-uniform $\mathrm{n}$-order hypergraph with average degree d. If it is triangle-free and linear, then $\alpha(\mathrm{H}) \geq \mathrm{nf}_{\mathrm{r}}(\mathrm{d})$.

Proof. We apply induction on $|\mathrm{V}|$, the number of vertices of $\mathrm{H}$. The result is trivial for $|V|=1$, since $f(0)=1$. Since the case $r=2$ is exactly what Shearer has given, we suppose that $r \geq 3$. 
For each $v \in H$, let $T=\left\{u_{1}, u_{2}, \ldots, u_{d(v)}\right\}$ be a claw of $v$. Since $H$ is $r$ uniform, linear and triangle-free, we have

$$
Q_{T}=d(v)+\sum_{i=1}^{d(v)}\left(d\left(u_{i}\right)-1\right)=\sum_{i=1}^{d(v)} d\left(u_{i}\right) .
$$

Let $\mathcal{T}_{v}$ be the set of all claws of $v$, then $\left|\mathcal{T}_{v}\right|=(\mathrm{r}-1)^{\mathrm{d}(v)}$. Therefore

$$
\sum_{\mathrm{T} \in \mathcal{T}_{v}} \mathrm{Q}_{\mathrm{T}}=\sum_{\mathrm{T} \in \mathcal{T}_{v}} \sum_{i=1}^{\mathrm{d}(v)} \mathrm{d}\left(\mathrm{u}_{\mathrm{i}}\right)=\sum_{\mathfrak{u} \in \mathfrak{n}(v)}(\mathrm{r}-1)^{\mathrm{d}(v)-1} \mathrm{~d}(\mathrm{u}),
$$

and

$$
\frac{1}{\left|\mathcal{T}_{v}\right|} \sum_{\mathrm{T} \in \mathcal{T}_{v}} \mathrm{Q}_{\mathrm{T}}=\sum_{\mathrm{u} \in \mathfrak{n}(v)} \frac{\mathrm{d}(\mathrm{u})}{\mathrm{r}-1} .
$$

We write $f(x)$ for $f_{r}(x)$ and set

$$
R_{T}(v)=1-(d(v)+1) f(d)+\left(d d(v)+d-r Q_{T}\right) f^{\prime}(d) .
$$

Then the average of $\mathrm{R}_{\mathrm{T}}(v)$ among $\mathrm{T} \in \mathcal{T}_{v}$ is

$$
\frac{1}{\left|\mathcal{T}_{v}\right|} \sum_{\mathrm{T} \in \mathcal{T}_{v}} \mathrm{R}_{\mathrm{T}}(v)=1-(\mathrm{d}(v)+1) \mathrm{f}(\mathrm{d})+(\mathrm{dd}(v)+\mathrm{d}) \mathrm{f}^{\prime}(\mathrm{d})-\mathrm{r} \sum_{\mathrm{u} \in \mathfrak{n}(v)} \frac{\mathrm{d}(\mathrm{u})}{\mathrm{r}-1} \mathrm{f}^{\prime}(\mathrm{d}) .
$$

Note that

$$
\frac{1}{n} \sum_{v \in V} \sum_{u \in N(v)} \frac{d(u)}{r-1}=\frac{1}{n} \sum_{v \in V} d^{2}(v) \geq d^{2}
$$

as $x^{2}$ is a convex function. Since $f^{\prime}(x)<0$, we have

$$
\frac{1}{n} \sum_{v \in V} \frac{1}{\left|\mathcal{T}_{v}\right|} \sum_{\mathrm{T} \in \mathcal{T}_{v}} R_{\mathrm{T}}(v) \geq 1-(\mathrm{d}+1) \mathrm{f}(\mathrm{d})+\left(\mathrm{d}^{2}+\mathrm{d}-\mathrm{rd}^{2}\right) \mathrm{f}^{\prime}(\mathrm{d})=0 .
$$

Hence there exists a vertex, say $v$, and a claw of $v$, say $T=\left\{\mathfrak{u}_{1}, \mathfrak{u}_{2}, \ldots, \mathfrak{u}_{\mathrm{d}(v)}\right\}$, such that $R(v) \geq 0$. Now by deleting $v$ and $u_{1}, u_{2}, \ldots, u_{d(v)}$, we obtain a new hypergraph $H^{\prime}$ with $n-d(v)-1$ vertices and $\frac{n d}{r}-Q_{T}$ edges. For an edge $e$ containing $v$, it contains $r \geq 3$ vertices, and we delete exactly two vertices from $e$, so $\mathrm{H}^{\prime}$ has some vertices. Note that the average degree $\overline{\mathrm{d}}$ of $\mathrm{H}^{\prime}$ is $\frac{n d-r Q_{T}}{n-d(v)-1}$. By induction hypothesis, we have

$$
\alpha(H) \geq(n-d(v)-1) f(\bar{d})=(n-d(v)-1) f\left(\frac{n d-r Q_{T}}{n-d(v)-1}\right) .
$$


Combining the facts that $\alpha(H) \geq 1+\alpha\left(H^{\prime}\right)$ and $f(x) \geq f(d)+f^{\prime}(d)(x-d)$ for all $x \geq 0$ as $f(x)$ is convex, we obtain

$$
\begin{aligned}
\alpha(H) & \geq 1+(n-d(v)-1) f\left(\frac{n d-r Q_{T}}{n-d(v)-1}\right) \\
& \geq 1+(n-d(v)-1) f(d)+\left(d d(v)+d-r Q_{T}\right) f^{\prime}(d) \\
& =n f(d)+R(v) \geq n f(d)
\end{aligned}
$$

completing the proof.

We now get an asymptotic form of $f_{r}(x)$ as $\frac{c}{x^{1 /(r-1)}}$ without knowing exact expression of $c=c(r)$ in hope of improving the old constant based on analysis of the algorithm as mentioned.

Lemma 21 Let $\mathrm{r} \geq 3$ be an integer. Then

$$
\lim x \rightarrow \infty f_{r}(x)=\frac{c}{x^{1 /(r-1)}},
$$

where $\mathrm{c}=\mathrm{c}(\mathrm{r})$ is a positive constant.

Proof. Recall that a first order linear differential equation $\frac{d y}{d x}=p(x) y+q(x)$ has the unique solution of the form

$$
y=e^{\phi(x)}\left(y_{0}+\int_{x_{0}}^{x} q(t) e^{-\phi(t)} d t\right)
$$

satisfying $y_{0}=y\left(x_{0}\right)$, where $\phi(x)=\int_{x_{0}}^{x} p(t) d t$. From the differential equation that $f_{r}(x)$ satisfies, we set

$$
p(x)=-\frac{x+1}{(r-1) x^{2}-x}, \text { and } q(x)=\frac{1}{(r-1) x^{2}-x} .
$$

For $x_{0}=2$,

$$
\phi(x)=-\int_{2}^{x} \frac{t+1}{(r-1) t^{2}-t} d t=\ln \frac{c_{1} x}{[(r-1) x-1]^{\frac{r}{r-1}}}
$$

Hence

$$
e^{\phi(x)}=\frac{c_{1} x}{[(r-1) x-1]^{\frac{r}{r-1}}} \sim \frac{c_{2}}{\chi^{1 /(r-1)}} .
$$

Then we have

$$
\mathrm{q}(\mathrm{t}) \mathrm{e}^{-\phi(\mathrm{t})} \sim \frac{1}{\mathrm{c}_{2}(\mathrm{r}-1)} x^{1 /(\mathrm{r}-1)-2},
$$


implying that $c_{3}=\int_{2}^{\infty} \mathrm{q}(\mathrm{t}) \mathrm{e}^{-\phi(\mathrm{t})} \mathrm{dt}<\infty$, and $\int_{2}^{\mathrm{x}} \mathrm{q}(\mathrm{t}) \mathrm{e}^{-\phi(\mathrm{t})} \mathrm{dt}=\mathrm{c}_{3}+\mathrm{o}(1)$ as $x \rightarrow \infty$. Therefore,

$$
f_{r}(x)=e^{\phi(x)}\left(y_{0}+c_{3}+o(1)\right) \sim \frac{c}{x^{1 /(r-1)}},
$$

where $c=c_{2}\left(y_{0}+c_{3}\right)$ and $y_{0}=f_{r}(2)$ are positive constants.

Acknowledgement The authors thank Attila Kiss (Eötvös Loránd University) and the unknown referee for the useful comments. This research is supported in part by the National Science Foundation of China (No. 11371193).

\section{References}

[1] V. Abraham, Independence in hypergraphs. J. Indian Math. Soc. (N.S.), 78, 1-4 (2011) $1-7 . \Rightarrow 141$

[2] B. D. Acharya, Interrelations among the notions of independence, domination and full sets in a hypergraph, Nat. Acad. Sci. Lett. 13, 11 (1990) 421-422. $\Rightarrow$ 142

[3] G. Agnarsson, A. Egilsson, M. M. Halldórson, Vertex coloring acyclic digraphs and their corresponding hypergraphs, Discrete Appl. Math. 156, 10 (2008) 19181928. $\Rightarrow 142$

[4] G. Agnarsson, M. M. Halldórson, E. Losievskaja, SDP-based algorithms for maximum independent set problems on hypergraphs, in: Automata, Languages and Programming. Part I, Lecture Notes in Comput. Sci. 5555, Springer, Berlin, 2009, 12-23. $\Rightarrow 133,138,141$

[5] G. Agnarsson, M. M. Halldórson, E. Losievskaja, SDP-based algorithms for maximum independent set problems on hypergraphs, Theoret. Comp. Sci. 470 (2013) $1-9 . \Rightarrow 133,138,141$

[6] M. Ajtai, P. Erdős, J. Komlós and E. Szemerédi, On Turán theorem for sparse graphs, Combinatorica 1, 3 (1981) 313-317. $\Rightarrow 134$

[7] M. Ajtai, P. Erdős, J. Komlós, E. Szemerédi, A dense infinite Sidon sequence, European J. Combin. 2, 1 (1981) 1-11. $\Rightarrow 134$

[8] M. Ajtai, J. Komlós and E. Szemerédi, A note on Ramsey numbers, J. Combin. Theory Ser. A 29 (1980) 354-360. $\Rightarrow 134$

[9] A. Alon, A fast and simple randomized parallel algorithm for the maximal independent set problem, J. Algorithms 7, 4 (1986) 567-583. $\Rightarrow 133,138$

[10] N. Alon, P. Frankl, H. Huang, V. Rödl, A. Ruciński, Large matchings in uniform hypergraphs and the conjectures of Erdős and Samuels, J. Combin. Theory Ser. A 119, 6 (2012) 1200-1215. $\Rightarrow 133,138,142$

[11] N. Alon, J. H. Spencer, The Probabilistic Method, Wiley-Interscience, New York, 1992. $\Rightarrow 134$ 
[12] A. Alon, A. Uri, Y. Azar, Independent sets in hypergraphs with applications to routing via fixed paths. In: Randomization, approximation, and combinatorial optimization (Berkeley, CA, 1999), Lecture Notes in Comput. Sci. 1671, Springer, Berlin, 1999, 16-27. $\Rightarrow 141$

[13] N. Alon, R. Yuster, On a hypergraph matching problem, Graphs Comb. 21 (2005) $377-384 . \Rightarrow 142$

[14] E. Angel, R. Campigotto, C. Laforest, A new lower bound on the independence number of graphs, Discrete Appl. 161, 6 (2013) 847-852. $\Rightarrow 134$

[15] G. Ausiello, A. D'Atri, D. Saccà, Minimal representation of directed hypergraphs, SIAM J. Comput. 15 (1986) 418-431. $\Rightarrow 139$

[16] J. Bailey, T. Manoukian, K. Ramamohanaro, A fast algorithm for computing hypergraph transversals and its application in mining emerging patterns, in: Proc. of the Third IEEE Int.l Conf. on Data Mining (ICDM03), 2003, 485-488. $\Rightarrow 142$

[17] J. Balogh, J. Butterfield, P. Hu, J. Lenz, D. Mubayi, On the chromatic thresholds of hypergraphs arXiv:1103.1416, 2013, 37 pages. $\Rightarrow 142$

[18] J. Balogh, R. Morris, W. Samotij, Independent sets in hypergraphs, arXiv:1204.6530, 2013, 42 pages. $\Rightarrow 136$

[19] Zs. Baranyai, On the factorization of the complete uniform hypergraph, in: A. Hajnal, R. Rado, V. T. Sós (Eds.), Infinite and Finite Sets, Proc. Coll. Keszthely, 1973, North-Holland, Amsterdam, Netherlands, 1975, pp. 91-108. $\Rightarrow 142$

[20] S. Behrens, C. Erbes, M. Ferrara, S. G. Hartke, B. Reiniger, H. Spinoza, C. Tomlinson, New results on degree sequences of uniform hypergraphs. Electron. J. Combin. 20, 4 (2013) \#P14, 18 pages. $\Rightarrow 139$

[21] M. Behrisch, A. Coja-Oghlan, M. Kang, The asymptotic number of connected d-uniform hypergraphs, Combin. Probab. Comput. 23, 3 (2014) 367-385. $\Rightarrow 139$

[22] C. Berge, The Theory of Graphs and its Applications, Wiley, London, $1964 . \Rightarrow$ 137

[23] E. Berger, R. Ziv, A note on the edge cover number and independence number in hypergraphs, Discrete Math. 308, 12 (2008) 2649-2654. $\Rightarrow 141$

[24] C. Bertram-Kretzberg, H. Lefmann, The algorithmic aspects of uncrowded hypergraphs. SIAM J. Comput. 29, 1 (1999) 201-230. $\Rightarrow 142$

[25] T. Biedl, E. D. Demaine, C. A. Duncan, R. Fleischer, S. G. Kobourov, Tight bounds on maximal and maximum matchings, Discrete Math. 285 (2004) 7-15. $\Rightarrow 141$

[26] V. Blinovsky, Fractional matching in hypergraphs, arXiv:1311.2671, 2013, 6 pages. $\Rightarrow 141$

[27] B. Bollobás, D. E. Daykin, P. Erdős, Sets of independent edges of a hypergraph, Quart. J. Math. Oxford Ser. (2) 27, 1 (1976), 25-32. $\Rightarrow 141$

[28] A. Bonato, J. I. Brown, D. Mitsche, P. Pralat, Independence densities of hypergraphs, arXiv:1308.2837, 2013, 16 pages. $\Rightarrow 141$ 
[29] A. Bonato, J. I. Brown, D. Mitsche, P. Pralat, Independence densities of hypergraphs, Europ. J. Combin. 40 (2014) 124-136. $\Rightarrow 141$

[30] R. Boppana, M. M. Halldórson, Approximating maximum independent set by excluding subsets, BIT, 32 (1992) 160-196. $\Rightarrow 133,138$

[31] M. Bordewich, M. Dyer, M. Karpiński, Path coupling using stopping times and counting independent sets and colorings in hypergraphs Random Structures Alg. 32, 3 (2008) 375-399. $\Rightarrow 141$

[32] E. Boros, K. Elbassioni, V. Gurvich, L. Khachiyan,Generating maximal independent sets for hypergraphs with bounded edge-intersections. In: LATIN 2004: Theoretical informatics, Lecture Notes in Comput. Sci. 2976, Springer, Berlin, 2004, 488-498. $\Rightarrow 141$

[33] E. Boros, V. Gurvich, K. Elbassioni, L. Khachiyan, An efficient incremental algorithm for generating all maximal independent sets in hypergraphs of bounded dimension. Parallel Process. Lett. 10, 4 (2000) 253-266. $\Rightarrow 141$

[34] M. Borowiecki, D. Michalak, The independence graphs of hypergraphs and middle graphs, Discuss. Math. 7 (1985) 31-37. $\Rightarrow 141$

[35] Cs. Bujtás, Zs. Tuza, Uniform mixed hypergraphs: The possible numbers of colors, Graphs Combin., 24 (2008) 1-12. $\Rightarrow 142$

[36] Y. Caro, New results on the independence number, Technical Report, 1979, TelAviv University. $\Rightarrow 134$

[37] Y. Caro, A. Hansberg, New approach to the k-independence number of a graph. Electron. J. Combin. 20, 1 (2013) \#P33, 17 pages. $\Rightarrow 135$

[38] Y. Caro, Zs. Tuza, Improved lower bounds on k-independence, J. Graph Theory 15 (1991) 99-107. $\Rightarrow 135$

[39] R. D. Carr, G. Lancia, S. Istrail, C. Genomics, Branch-and-Cut algorithms for independent set problems: Integrality gap and an application to protein structure alignment. SAND Report SAND2000-2171, Sandia National Laboratories, 2000. $\Rightarrow 142$

[40] G. Chartrand, S. Schuster, On the independence number of complementary graphs, Trans. New York Acad. Sci., Series II 36, 3 (1974) 247-251. $\Rightarrow 139$

[41] M. Chellali, O. Favaron, A. Hansberg, L. Volkmann, k-domination and kindependence in graphs: a survey, Graphs Combin. 28, 1 (2012) 1-55. $\Rightarrow 135$, 137

[42] M. Chellali, N. J. Rad, On k-independence critical graphs. Australas. J. Combin. 53 (2012) 289-298. $\Rightarrow 135$

[43] E. J. Cockayne, S. T. Hedetniemi, R. Laskar, Gallai theorems for graphs, hypergraphs and set systems, Discrete Math. 72 (1988) 35-47. $\Rightarrow 142$

[44] B. Csaba, T. A. Pick, A Shokoufandeh, A note on the Caro-Tuza bound on the independence number of uniform hypergraphs, Australas. J. Combin. 52 (2012) $235-242 . \Rightarrow 135$

[45] J. Cutler, A. J. Radcliffe, Hypergraph independent sets, Combin. Probab. Comput. 22, 1 (2013) 9-20. $\Rightarrow 141$ 
[46] B. C. Dean, S. M. Hedetniemi, S. T. Hedetniemii, J. Lewis, A. McRae, Matchability and k-maximal matchings. Discrete Math. 159, 1 (2011) 15-22. $\Rightarrow 141$

[47] A. Dudek, A. Frieze, A. Ruciński, M. Šileikis, Approximate counting of regular hypergraphs, Inf. Processing Letters, 113, 1921 (2013) 785-788. $\Rightarrow 139,141$

[48] K. Dutta, D. Mubayi, C. R. Subramanian, New lower bounds for the independence number of sparse graphs and hypergraphs. SIAM J. Discrete Math. 26, 3 (2012) 1134-1147. $\Rightarrow 138$

[49] J. Edmonds, Paths, trees, and flowers, Canadian J. Math. 17 (1965) 449-467. $\Rightarrow 133,138,141$

[50] J. Edmonds, Maximal matching and a polyhedron with 0, 1-vertices, J. Research Nat. Bureau Standards B-69 (1965) 125-130. $\Rightarrow 133,138,141$

[51] A. Eustis, Hypergraph Independence Numbers, PhD Thesis. University of California, San Diego. 2013. 123 pages. $\Rightarrow 138$

[52] A. Eustis, J. Verstraëte, On the independence number of Steiner systems, Combinatorics, Prob. Comp. 22, 2 (2013) 241-252. $\Rightarrow 138$

[53] S. Fajtlowicz, Independence, clique size and maximum degree, Combinatorica 4 (1984), 35-38. $\Rightarrow 136$

[54] O. Favoron, A. Hansberg, L. Volkmann, On k-domination and minimum degree in graphs, J. Graph Theory 57, 1 (2008) 33-40. $\Rightarrow 135,137$

[55] A. Frank, T. Király, Z. Király, On the orientation of graphs and hypergraphs, Discrete Appl. Math 131, 2 (2003) 385-400. $\Rightarrow 140,142$

[56] P. Frankl, Improved bounds for Erdös matching conjecture, J. Combin. Theory Ser. A 120, 5 (2013) 1068-1072. $\Rightarrow 141$

[57] P. Frankl, T. Luczak, K.Mieczkowska, On matchings in hypergraphs. Electron. J. Combin. 19, 2 (2012), \#P42, 5 pages. $\Rightarrow 141$

[58] P. Frankl, V. Rödl, Some Ramsey-Turn type results for hypergraphs. Combinatorica 8, 4 (1988) 323-332. $\Rightarrow 142$

[59] P. Frankl, V. Rödl, The uniformity lemma for hypergraphs, Graphs Combin. 8 (1992) 309-312. $\Rightarrow 142$

[60] M. Frieze, On the independence number of random graphs, Discrete Math. 81, 2 (1990) $171-175$. $\Rightarrow 136$

[61] Z. Füredi, Matchings and covers in hypergraphs, Graphs Combin. 4 (1988) 115206. $\Rightarrow 141$

[62] Z. Füredi, The number of maximal independent sets in connected graphs, $J$. Graph Theory, 11, 4 (1995) 463-470. $\Rightarrow 137$

[63] Z. Füredi, Linear trees in uniform hypergraphs, Europ. J. Combin. 35 (2014) 264-272. $\Rightarrow 142$

[64] Z. Füredi, M. Ruszinkó, Uniform hypergraphs containing no grids, Adv. Math. 240 (2013) 302-324. $\Rightarrow 142$

[65] H. M. Gabow, An efficient implementation of Edmonds' algorithm for maximum matching on graphs, J. ACM 23, 2 (1976) 221-234. $\Rightarrow 141$ 
[66] H. M. Gabow, Data structures for weighted matchings and nearest nearest common ancestors with linking, in: Proc. 1st Annual ACM-SIAM Symp. Discrete Algorithms 1990, 434-443. $\Rightarrow 141$

[67] T. Gallai, Über extreme Punkt- und Kantenmengen, Ann. Univ. Sci. Budapest. de Rolando Eötvös Nominatae, Sect. Math. 2 (1959) 133-138. $\Rightarrow 139$

[68] G. Gallo, G. Longo, S. Nguyen, S. Pallottino, Directed hypergraphs and applications, Discrete Appl. Math 42, 2-3 (1993) 177-201. $\Rightarrow 140,142$

[69] S.. Gaspers, D. Kratsch, M. Liedloff, Independent sets and bicliques in graphs, in: H. Broersma, T. Erlebach, T. Friedetzky, D. Paulusma (eds.) Graph-Theoretic Concepts in Computer Science (Int. Workshop WG2008, Durham, UK, June 30, 2008-July 2, 2008), Lecture Notes in Comput. Sci. 5344, Springer, 2008, $171-182 . \Rightarrow 136$

[70] C. Greenhill, The complexity of counting colourings and independent sets in sparse graphs and hypergraphs. Comput. Complexity 9, 1 (2000) 52-72. $\Rightarrow 141$

[71] C. Greenhill, A. Rucinski, N. C. Wormald, Random hypergraph processes with degree restrictions, Graphs Combin. 20 (2004) 319-332. $\Rightarrow 141$

[72] J. Griggs, Lower bounds on the independence number in terms of the degrees, J. Comb. Theory, Ser. B 34 (1983) 22-39. $\Rightarrow 134,145$

[73] J. L. Gross, J. Yellen, P. Zhang, Handbook of Graph Theory, CRC Press, Boca Raton, FL, 2014. $\Rightarrow 133,138$

[74] D. Gunopolus, R. Khardon, H. Mannila, H. Toivonen, Data mining, hypergraph traversals, and machine learning. Proc. PODS'97, 1997, pp. 209-2016. $\Rightarrow 142$

[75] M. M. Halldórson, Approximations of weighted independent set and hereditary subset problems, J. Graph Algorithms Appl. 4, 1 (2000) 1-16. $\Rightarrow 136$

[76] M. M. Halldórson, E. Losievskaja, Independent sets in bounded-degree hypergraphs, Discrete Appl. Math 157, 8 (2009) 1773-1786. $\Rightarrow 141$

[77] J. Han, Near perfect matchings in k-uniform hypergraphs, arXiv:1404.1136, 2014, 7 pages. $\Rightarrow 141$

[78] H. Hàn, Y. Person, M. Schaht, On perfect matchings in uniform hypergraphs with large minimum vertex degree, SIAM J. Discrete Math. 23, 2 (2009) 732748. $\Rightarrow 141,142$

[79] A. Hansberg, R. Pepper, On k-domination and j-independence in graphs. Discrete Appl. Math 161, 10-11 (2013) 1472-1480. $\Rightarrow 135,136,137$

[80] J. Harant, A lower bound on independence number of a graph, Discrete Math. 188, 13 (1998) 239243. $\Rightarrow 134$

[81] J. Harant, A lower bound on independence in terms of degrees, Discrete Appl. Mathematics 159, $10(2011)$ 966-970. $\Rightarrow 134,135$

[82] J. Harant, A. Pruchnewski, M. Voigt, On dominating sets and independent sets of graphs, Combinatorics, Prob. Comp. 8, 6 (1999) 547-553. $\Rightarrow 137$

[83] J. Harant, D. Rautenbach, Independence in connected graphs. Discrete Appl. Math 159, 1 (2011) 79-86. $\Rightarrow 135$

[84] J. Harant, I. Schiermeyer, On the independence number of a graph in terms of order and size, Discrete Math., 232, 1-3 (2001) 131-138. $\Rightarrow 135$ 
[85] J. Harant, I. Schiermeyer, A lower bound on the independence number of a graph in terms of degrees. Discuss. Math. Graph Theory 26, 3 (2006), 431-437. $\Rightarrow 135$

[86] M. A. Henning, C. Löwenstein, D. Rautenbach, Independent sets and matchings in subcubic graphs. Discrete Math., 312, 11 (2012) 1900-1910. $\Rightarrow 141$

[87] M. A. Henning, C. Löwenstein, J. Southey, A. Yeo. A new lower bound on the independence number of a graph and applications, Electron. J. Comb. 21, 1 (2014) \#P1.38. $\Rightarrow 136$

[88] M. A. Henning, A. Yeo, Tight lower bounds on the size of a maximum matching in a regular graph. Graphs Combin. 3, 6 (2007) 647-657. $\Rightarrow 141$

[89] M. A. Henning, A. Yeo, Lower bounds on the size of maximum independent sets and matchings in hypergraphs of rank three. J. Graph Theory, 72, 1-2 (2013) $220-245 . \Rightarrow 135,141,142$

[90] T. Hofmeister, H. Lefmann, Approximating maximum independent sets in uniform hypergraphs. In: Mathematical Foundations of Computer Science, 1998, Brno, Lecture Notes in Comput. Sci. 1450, Springer, Berlin, 1998, 562-570. $\Rightarrow$ 141

[91] J. E. Hopcroft, R. M. Karp, An $n^{5 / 2}$ algorithm for maximum matchings in bipartite graphs. SIAM J. Comput., 2 (1973) 225-231. $\Rightarrow 141$

[92] H. Huang, P.-S. Loh, B. Sudakov, The size of a hypergraph and its matching number, Combin. Probab. Comput. 21, 3 (2012) 442-450. $\Rightarrow 141,142$

[93] D. S. Johnson, M. Yannakakis, On generating all maximal independent sets, Inf. Processing Letters 27, 3 (1988) 119-123. $\Rightarrow 133,138,141,142$

[94] T. Johnston, L. Lu, Turn problems on non-uniform hypergraphs, arXiv:1301.1870, 2013. $\Rightarrow 142$

[95] T. Johnston, L. Lu, Strong jumps and Lagrangians of non-uniform hypergraphs, arXiv: $1403.1220,2014 . \Rightarrow 142$

[96] B. K. Jose, Zs. Tuza, Hypergraph domination and strong independence. Appl. Anal. Discrete Math. 3, 2 (2009) 347-358. $\Rightarrow 142$

[97] E. Jucovič, F. Olejník, On chromatic and achromatic numbers of uniform hypergraphs, Časopis Pěstováni Matematiky, 99 (1974) 123-130. $\Rightarrow 139,142$

[98] A. Kako, T. Ono, T. Hirata, M. M. Halldórson, Approximation algorithms for the weighted independent set problem in sparse graphs, Discrete Appl. Math 157, 4 (2009) 617-626. $\Rightarrow 136$

[99] M. Karonski, T. Luczak, The number of connected sparsely edged uniform hypergraphs, Discrete Math. 171, 1-3 (1997) 153-167. $\Rightarrow 142$

[100] R.M. Karp, U.V. Vazirani, V.V. Vazirani, An optimal online algorithm for optimal bipartite matching, in: Proceedings of the Twenty-second Annual ACM Symposium on Theory of Computing (STOC '90), ACM, New York, NY, 1990, $352-358 . \Rightarrow 141$

[101] R. M. Karp, A. Widgerson, A fast parallel algorithm for the maximal independent set problem, J. ACM 32, 4 (1985) 762-773. $\Rightarrow 133,138$ 
[102] G. O. H. Katona, Continuous versions of some extremal hypergraph problems, in: Combinatorics (Keszthely, Hungary, 1976), Coll. Math. Soc. J. Bolyai 18 (Math. Soc. J. Bolyai, Budapest, 1978), 653-678. $\Rightarrow 142$

[103] G. O. H. Katona, Continuous versions of some extremal hypergraph problems II, Acta Math. Acad. Sci. Hungar. 35 (1980) 67-77. $\Rightarrow 142$

[104] P. Keevash, F. Knox, R. Mycroft, Polynomial-time perfect matchings in dense hypergraphs, arXiv:1307.2608, 2013. 62 pages. $\Rightarrow 141$

[105] P. Keevash, R. Mycroft, A geometric theory for hypergraph matching, arXiv:1108.1757, 2013, 101 pages. $\Rightarrow 141$

[106] P. Keevash, B. Sudakov, On a hypergraph Turán problem of Frankl, Combinatorica 25, 6 (2005) 673-706. $\Rightarrow 142$

[107] P. Kelsen, On the parallel complexity of computing a maximal independent set in a hypergraph. In: Proc. Twenty-fourth Annual ACM Symp. Theory Comp. (STOC'92), ACM New York, NY, 1992, 339-350. $\Rightarrow 138,142$

[108] L. Khachiyan, E. Boros, V. Gurvich, K. Elbassioni, Computing many maximal independent sets for hypergraphs in parallel, Parallel Process. Lett. 17, 2 (2007) $141-152 . \Rightarrow 141$

[109] I. Khan, Perfect matchings in 3-uniform hypergraphs with large vertex degree. SIAM J. Discrete Math. 27, 2 (2013) 1021-1039. $\Rightarrow 141$

[110] M. A. Khan, S. , K. K. Kayibi, Scores, inequalities and regular hypertournaments, Math. Inequal. Appl. 15, 2 (2012), 343-351. $\Rightarrow 139$

[111] Y. Kohayakawa, V. Rödl, J. Skokan, Hypergraphs, quasi-randomness, and conditions for regularity, J. Combin. Theory Ser. A 97, 2 (2002) 307-352. $\Rightarrow 142$

[112] V. Kolmogorov, V. Blossom, A new implementation of a minimum cost perfect matching algorithm, Math. Programming Pomp. 1 (2009) 43-67. $\Rightarrow 141$

[113] D. König, Graphs and matrices (Hungarian), Matematikai és Fizikai Lapok 38 (1931) $116-119 . \Rightarrow 141$

[114] A. Kostochka, D. Mubayi, J. Verstraëte, On independent sets in hypergraphs, Random Structures Algorithms 44, 2 (2014) 224-239. $\Rightarrow 142$

[115] M. Krivelevich, Approximate set covering in uniform hypergraphs, J. Algorithms 25, 1 (1997) 118-143. $\Rightarrow 142$

[116] M. Krivelevich, R. Nathaniel, B. Sudakov, Approximating coloring and maximum independent sets in 3-uniform hypergraphs, J. Algorithms 41, 1 (2001) 99-113. $\Rightarrow 142$

[117] D. Kühn, D. Loose, Hamilton cycles in 3-uniform hypergraphs of high minimum degree, J. Comb. Theory, Ser. B 96, 6 (2006) 767-821. $\Rightarrow 142$

[118] D. Kühn, D. Osthus, A. Treglown, Matchings in 3-uniform hypergraphs, $J$. Combinatorial Theory Series B 103 (2013) 291-305. $\Rightarrow 141$

[119] D. Kühn, D. Osthus, T. Townsend, Fractional and integer matchings in uniform hypergraphs, Europ. J. Combin. 38 (2014) 83-96. $\Rightarrow 141$

[120] R. Laskar, B. Auerbach, On complementary graphs with no isolated vertices, Discrete Math. 24, 2 (1978) 113-118. $\Rightarrow 140$ 
[121] E. L. Lawler, J. K. Lenstra, A. H. G. Rinnooy Kan, Generating all maximal independent sets: NP-hardness and polynomial-time algorithms, SIAM J. Comput. 9, 3 (1980), 558-565. $\Rightarrow 133,138$

[122] V. V. Lepin, An algorithm for finding the independence number of a recursively generated hypergraph (Russian), Dokl. Nats. Akad. Nauk Belarusi 49, 2 (2005), $22-25,125 . \Rightarrow 141$

[123] Y. Li, C. Rousseau, On book-complete graph Ramsey numbers, J. Comb. Theory, Ser. B 68, 1 (1996) 36-44. $\Rightarrow 142$

[124] Y. Li, C. Rousseau, W. Zang, Asymptotic upper bound for Ramsey functions, Graphs Combin., 17 (2001) 123-128. $\Rightarrow 142$

[125] Y. Li, W. Zang, Differential methods for finding independent sets in hypergraphs, SIAM J. Discrete Math. 20 (2006) 96-104. $\Rightarrow 141,142$

[126] E. Losievskaja, Approximation Algorithms for Independent Set Problems on Hypergraphs, PhD Dissertation, School of Computer Science Reykjavik University, Reykyavik, 2009, XV + 80 pages. $\Rightarrow 133,138,141$

[127] L. Lovász, M. D. Plummer, Matching Theory, North Holland, Amsterdam, 1986. $\Rightarrow 141$

[128] M. Luby, A simple parallel algorithm for the maximal independent set problem, SIAM J. Comput. 15, 1 (1976) 1036-1053. $\Rightarrow 133,138$

[129] T. Luczak, E. Szymańska, A parallel randomized algorithm for finding a maximal independent set in a linear hypergraph, J. Algorithms 25, 2 (1997) 311-320. $\Rightarrow 142$

[130] D. Maier, Minimum covers in the relational data base model, J. Assoc. Comp. Mach. 27 (1980) 664-674. $\Rightarrow 142$

[131] K. Markström, A. Ruciński, Perfect matchings (and Hamilton cycles) in hypergraphs with large degrees, Europ. J. Combin. 32, 5 (2011) 677-687. $\Rightarrow 141$

[132] S. Micali, V.V. Vazirani, An $\mathrm{O}(\sqrt{\mathrm{V}} \mathrm{E})$ algorithm for finding maximum matching in general graphs, Proc. 21st Annual IEEE Symposium on Foundations of Computer Science (1980) 17-27. $\Rightarrow 141$

[133] K. Mulmuley, U.V. Vazirani, V.V. Vazirani, Matching is as easy as matrix inversion, Combinatorica 7, 1 (1987) 105-114. $\Rightarrow 141$

[134] V. Nikiforov, An analytic theory of extremal hypergraph problems, arXiv:1305.1073, 2013, 31 pages. $\Rightarrow 142$

[135] F. Olejník, On total matching numbers and total covering numbers for k-uniform hypergraphs. Math. Slovaca 34, 3 (1984), 319-328. $\Rightarrow 141$

[136] F. Olejník, On edge independence numbers and edge covering numbers of kuniform hypergraph, Math. Slovaca 39, 1 (1989) 21-26. $\Rightarrow 138,139,140$

[137] L. Özkahiya, M. E. Young, Anti-Ramsey number of matchings in hypergraphs, Discrete Math., 313, 20 (2013), 2359-2364. $\Rightarrow 141$

[138] S. Pirzada, Hypertournaments-scores, losing scores, total scores and degrees. $J$. Combin. Math. Combin. Comput. 84 (2013) 99-112. $\Rightarrow 139$

[139] S. Pirzada, G. Zhou, On k-hypertournament losing scores, Acta Univ. Sapientiae, Informatica 2, 1 (2010) 5-9. $\Rightarrow 139$ 
[140] S. Pirzada, G. Zhou, A. Iványi, Score lists in multipartite hypertournaments, Acta Univ. Sapientiae, Informatica 2, 2 (2010) 184-193. $\Rightarrow 139$

[141] K. Plociennik, Approximating independent set and coloring in random uniform hypergraphs. In: Mathematical Foundations of Computer Science (2008), Lecture Notes in Comput. Sci. 5162, Springer, Berlin, 2008, 539-550. $\Rightarrow 141$

[142] M. D. Plummer, Matching theory-a sampler: from Dénes König to the present, Discrete Math. 100 (1992) 172-219. $\Rightarrow 141$

[143] A. Pruchnewski, On the domination number of graphs, Discrete Math., 251, 1-3 (2002) 129-136. $\Rightarrow 137$

[144] J. Qian, Enumeration of unlabeled uniform hypergraphs, Electronic J. Combin 20, 1 (2013), P46, 10 pages $\Rightarrow 139$

[145] J. Qian, Enumeration of unlabeled uniform hypergraphs. Discrete Math. 326 (2014), 66-74. $\Rightarrow 139$

[146] V. Rödl, A. Ruciśki, M. Schacht, E. Szemerédi, A note on perfect matchings in uniform hypergraphs with large minimum collective degree, Comment. Math. Univ. Carolin. 49, 4 (2008) 633-636. $\Rightarrow 141$

[147] V. Rödl, A. Ruciński, E. Szemerédi, Perfect matchings in uniform hypergraphs with large minimum degree, Europ. J. Combin. 27 (2006) 1333-1349. $\Rightarrow 141$

[148] V. Rödl, A. Ruciński, E. Szemerédi, Perfect matchings in large uniform hypergraphs with large minimum collective degree, J. Combin. Theory Ser. A 116, 3 (2009), 613-636. $\Rightarrow 141$

[149] S. Sakai, M. Mitsunori, K. Yamazaki, A note on greedy algorithms for the maximum weighted independent set problem. Discrete Appl. Math 126, 2-3 (2003) $313-322 . \Rightarrow 136$

[150] S. M. Selkow, A probabilistic lower bound on the independence number of graphs. Discrete Math. 132 (1994) 363-365. $\Rightarrow 134,135$

[151] H. Shachnai, A. Srinivasan, Finding large independent sets in graphs and hypergraphs. SIAM J. Discrete Math. 18, 3 (2004/05) 488-500. $\Rightarrow 141$

[152] J. Shearer, A note on the independence number of triangle-free graphs, Discrete Math. 46 (1983) 83-87. $\Rightarrow 134,136,141,145$

[153] J. Spencer, Turán's theorem for k-graphs, Discrete Math. 2 (1972) 183-186. $\Rightarrow$ 136

[154] E. Szymańska, The complexity of 2-coloring and strong coloring in uniform hypergraphs of high minimum degre, Discrete Math. Theor. Comput. Sci. 15, 3 (2013) 121-137. $\Rightarrow 141,142$

[155] R. E. Tarjan, A. E. Trojanowski, Finding a maximum independent set, SIAM J. Comput. 6, 3 (1977) 537-546. $\Rightarrow 133,138,141$

[156] T. Thiele, A lower bound on the independence number of arbitrary hypergraphs, J. Graph Theory 30, 3 (1999) 213-221. $\Rightarrow 135$

[157] A. Treglown, Y. Zhao, Exact minimum degree thresholds for perfect matchings in uniform hypergraphs, J. Combin. Theory Ser. A 119, 7 (2012) 1500-1522. $\Rightarrow 141,142$ 
[158] A. Treglown, Y. Zhao, Exact minimum degree thresholds for perfect matchings in uniform hypergraphs II., J. Combin. Theory Ser. A 120, 7 (2013) 1463-1482. $\Rightarrow 141,142$

[159] P. Turán, On the theory of graphs, Colloq. Math. 3 (1954) 19-30. $\Rightarrow 133,134$

[160] Zs. Tuza, Extensions of Gallai's graph covering theorems for uniform hypergraphs, J. Combin. Theory, Series B 52, 1 (1991) 92-96. $\Rightarrow 139,142$

[161] V. V. Vazirani, A theory of alternating paths and blossoms for proving correctness of the $\mathrm{O}(\sqrt{V} E)$ maximum matching algorithm, Combinatorica 14, 1 (1994) $71-109 . \Rightarrow 133$

[162] V. V. Vazirani, An improved definition of blossoms and a simpler proof of the MV matching algorithm, arXiv:1210.4594, 2013, 32 pages. $\Rightarrow 133$

[163] A. Vietri, The complexity of arc-colorings for directed hypergraphs, Discrete Appl. Math 143,1-3 (2004) 266-271. $\Rightarrow 140$

[164] C. Wang, G. Zhou, Note on the degree sequences of k-hypertournaments, Note on the degree sequences of k-hypertournaments, Discrete Math. 308, 11 (2008) $2292-2296$. $\Rightarrow 139$

[165] V. Wei, A lower bound on the stability number of a simple graph, Bell Laboratories Technical Memorandum, 1981, No. 81-11217-9. $\Rightarrow 134$

[166] E. W. Weisstein, Independent Edge Set. Downloaded 9 May 2014. $\Rightarrow 133$

[167] Wikipedia, Independent Set. Downloaded 9 May 2014. $\Rightarrow 133,138$

[168] R. Yuster, Finding and counting cliques and independent sets in r-uniform hypergraphs. Inf. Processing Letters 99, 4 (2006) 130-134. $\Rightarrow 141$

[169] R. Yuster, Maximum matching in regular and almost regular graphs, Algorithmica 66, 1 (2013) 87-92. $\Rightarrow 141,142$

[170] G. Zhou, Y. Li, Independence numbers of hypergraphs with sparse neighborhoods, Europ. J. Combin. 25, 3 (2004) 355-362. $\Rightarrow 134,140,141,142$

[171] G. Zhou, S. Pirzada, Degree sequence of oriented k-hypergraphs, J. Appl. Math. Comput. 27, 1-2 (2008) 149158. $\Rightarrow 139$

[172] G. Zhou, T. Yao, K. Zhang, On score sequences of k-hypertournaments, Europ. J. Combin. 21, 8 (2000) 993-1000. $\Rightarrow 139$

Received: January 15, 2014・Revised: May 11, 2014 